Cahiers de recherches médiévales

\title{
Le choix de la prose
}

\section{Emmanuèle Baumgartner}

\section{(2) OpenEdition}

Journals

Édition électronique

URL : https://journals.openedition.org/crm/1322

DOI : $10.4000 / \mathrm{crm} .1322$

ISSN : 1955-2424

Éditeur

Honoré Champion

Édition imprimée

Date de publication : 30 octobre 1998

Pagination : 7-13

ISSN : 1272-9752

Référence électronique

Emmanuèle Baumgartner, « Le choix de la prose », Cahiers de recherches médiévales [En ligne], 5 | 1998, mis en ligne le 01 octobre 2007, consulté le 15 décembre 2022. URL : http://journals.openedition.org/ crm/1322 ; DOl : https://doi.org/10.4000/crm.1322

Ce document a été généré automatiquement le 15 décembre 2022.

Tous droits réservés 


\title{
Le choix de la prose
}

\author{
Emmanuèle Baumgartner
}

Le verbe familier s'est fait prose ; le verbe
transcendant s'est incarné dans le vers.
L'un a discouru, l'autre a chanté.
Lamartine (Cours familier de Littérature, IV

Entretien)

1 Le développement d'une prose littéraire en langue vernaculaire est l'un des événements majeurs de la création littéraire au XIII ${ }^{\mathrm{e}}$ siècle, même si le vers narratif reste durant tout ce siècle au moins un concurrent sérieux, qui s'approprie de nouveaux genres comme le fabliau, le dit ou encore, avec Jean de Meun, la poésie scientifique et philosophique. Comme toute forme neuve ou appelée à un nouvel usage, la prose française a dû alors se situer sur l'échiquier littéraire et se délimiter un créneau. À en juger par les témoignages publiés par B. Woledge et H. P. Clive dans leur Répertoire des plus anciens textes en prose française ${ }^{1}$ et largement exploités depuis par la critique, le créneau qui a été alors choisi par les clercs, le manifeste qu'ils ont lancé, est l'exigence de "vérité » et l'affirmation, parfaitement sujette à caution, que la prose, liée à l'écrit, à la différence du vers voué aux aléas de la diffusion orale, est par excellence le médium qui peut garantir la véracité et l'authenticité du texte produit. C'est ce qu'on peut lire par exemple dans le prologue souvent cité de la première traduction de la Chronique dite du Pseudo-Turpin, datée des alentours de 1200 et composée selon B. Woledge et H. P. Clive à la demande de Yolande et Hugues de SaintPol, à partir d'un manuscrit latin légué par Baudouin VIII, comte de Flandre :

Voil comencier l'estoire si cum li bons enpereire Karlemaines en ala en Espanie por

la terra conquerra sor Sarrazins. Maintes genz en ont oï conter et chanter, mes n'est si mensongie non ço qu'il en dient et chantent cil jogleor ne cil conteor. Nus contes rimés n'est verais. Tot est menssongie ço qu'il en dient, quar il non seivent rien fors par oïr dire. Li bons Baudoins li cuens de Chainau si ama molt Karlemaine, ne ne voc unques croire chose que l'en chantast, ainz fit cercher totes les bones abaies de France e garder par toz les armaires por savoir si l'om i troveroit la veraie estoira, ni onques trover ne l'i porent li clerc. ${ }^{2}$ 
2 Suivent des considérations sur la quête et la découverte de la «bonne estoire », celle qu'a composée en Espagne au contact même des événements vécus «Turpins li bons arcevesques de Reins... qui avoc le bon enpereor fu e par jor e par nuit» et qui rappellent étrangement les déclarations plus anciennes de Benoît de Sainte-Maure dans le prologue du Roman de Troie.

3 Cette déclaration polémique, qui dénie aux "contes rimés» (aux romans ou plus sûrement ici aux chansons de geste) la faculté de dire le vrai et transfère par défaut (car le terme n'apparaît qu'en 1265 dans le Livre du Trésor de Brunetto Latini) cette faculté sur la prose et sur le livre, devient très vite et pour longtemps un véritable topos. On le trouve de manière attendue dans les prologues des chroniqueurs, mais il est également repris par certains auteurs de romans en prose. On connaît le passage célèbre du Perceval en prose dans lequel l'auteur, s'en prenant à Chrétien de Troyes et aux « autre troveor qui en ont trové por faire lor rimes plaisans ", déclare que lui ne relate rien d'autre «fors tant com au conte en monte et que Merlins en fist escrire a Blayse son maistre $»^{3}$ et souligne ainsi l'authenticité d'une prose débarrassée des ornements mensongers du vers et fidèle à sa source, le livre dicté par Merlin. Une formulation plus tardive et plus polémique encore est le prologue de la Chronique de Jean le Bel dans lequel le chroniqueur recommande au lecteur de lire «ce petit livre que j'ay commencé à faire, et laisse ung grand livre rimé que j'ay veu et leu, lequel aucun controuveur a mis en rime par grandes faintes et bourdes controuvées... $»^{4}$

Placer une œuvre littéraire profane, composée en français, sous le signe de la vérité, tenter ainsi de la faire passer du statut peu glorieux de "fable» à celui de texte " autorisé », n'est pas cependant une exigence qui daterait du XIII ${ }^{e}$ siècle et apparaitrait avec le choix de la prose. Mais peut-être faudrait-il préciser les sens que les écrivains médiévaux donnent au fil du temps à des termes comme verai, voir, verur. Lorsque Marie de France déclare aux vv. 19-20 du prologue de Guigemar «les contes que jo sai verais,/dunt li Bretun unt fait les lais,/vos conterai assez briefment ", lorsque, faisant sans doute écho à une déclaration liminaire, Thomas souligne à la fin de son Tristan (vv. 3133-3134) « dit ai tute la verur/si cum jo pramis al primur », verai, verur désignent une vérité d'ordre moral, l'enseignement que ces récits peuvent délivrer aux lecteurs et, chez Thomas, la cohérence interne du récit, plutôt que l'authenticité des histoires rapportées. Et n'est-il pas saugrenu que les fondements d'un discours sur la vérité de la prose se lisent en tête du Pseudo-Turpin, l'un des textes les plus fictionnels qu'ait produits le XIII ${ }^{e}$ siècle ? On peut se demander si le traducteur n'a pas précisément lancé ce brûlot, le mensonge véhiculé par le vers, opposé à la véracité d'une prose fondée sur des sources écrites, pour masquer le caractère fabuleux du texte qu'il présentait à un public décidé, dit-on, à s'instruire plus qu'à se divertir à la lecture de romans ${ }^{5}$; et spécialement à la lecture de cette matière de Bretagne à laquelle Jean Bodel a accolé de manière tenace l'étiquette de contes «vains et plaisans».

La trinité écriture-prose-vérité avait d'ailleurs de quoi séduire à plus d'un titre. Prétendre cerner de plus près la vérité d'une histoire/de l'Histoire par le choix de la prose est aussi pour des clercs en quête de sujets à traiter une façon de justifier la pratique de la réécriture de textes en vers, la production de tel ou tel rifacimento. On ne croira pas pour autant que ces clercs aient été assez stupides pour ne pas savoir que l'on peut aussi bien pratiquer le mensonge et même le «mentir vrai» en prose qu'en vers. On soupçonnera déjà certains d'entre eux, notamment ceux qui ne furent pas des 
professionnels de l'écriture, d'avoir vu dans la prose une forme qui exigeait moins de métier et/ou moins de travail que l'écriture en vers. Dans sa Chronique des ducs de Normandie, qui s'appuie sur les chroniques en prose latine de Dudon de Saint-Quentin et de Guillaume de Jumièges (Dudon faisant alterner prose et vers) Benoît de SainteMaure dit la difficulté qu'il éprouve à «comprendre» dans l'espace du couplet d'octosyllabes la complexité du latin :

Mais li latins dit et comprend

Od somme, od glose, ce m'est vis,

Ou rommanz ne puet estre mis

Choses moutes ; por ce m'est gref. ${ }^{6}$

6 À l'époque où il écrit, il devait être inconcevable pour un écrivain de métier, ce qu'il fut vraisemblablement, de se donner cette facilité, écrire en prose, emprunter, pour citer Brunetto Latini ${ }^{7}$ cette voie «large et pleniere ", pour déployer plus aisément la grande rhétorique du discours argumentatif ou pour conter sur le mode épique les combats, historiquement attestés, des Normands contre leurs multiples adversaires. Si, beaucoup plus tard, Christine de Pizan compose en prose la partie de son Livre de Mutacion de Fortune consacrée aux Juifs, c'est, dit-elle, par ce qu'elle est malade et que "pour mon ovrage haster,/Mettray la prose en la maniere/Que mot a mot l'escri plainiere", ajoutant que «Et qui de bien rimer se charge/Ce n'est mie petites charges,/Et par especial, histoires/Abriger en paroles voires. ${ }^{8}$

7 Au début du XIII ${ }^{e}$ siècle, la coupure entre la prose vérité et le vers mensonger est d'ailleurs loin d'être évidente. Lorsqu'il compose vers 1213-1214 un texte en prose appelé par tradition depuis les travaux de Paul Meyer l'Histoire ancienne jusqu'à César, l'auteur, dans son prologue en vers, martèle en octosyllabes son intention de rapporter dans toute sa vérité et en suivant pas à pas la «letre» (le texte latin) l'histoire du monde :

\footnotetext{
L'uevre iert mout bone e delitable

E d'estoire sans nulle fable,

Por ce iert plaisans e creüe.

La verité fait bon entendre,

Oïr, retenir e aprendre

Qui verité aime e retient

As comans Damedeu se tient.

Je n'i veull fors verité dire. ${ }^{9}$
}

8 Qui plus est, du moins dans la première rédaction du texte, s'oberve une répartition intéressante entre le récit proprement dit, en prose, et des passages en vers qui donnent un commentaire le plus souvent didactique et moral des faits évoqués ${ }^{10}$. D'un côté donc la vérité historique, du moins ce qui est reçu comme tel, s'énonce en prose ; de l'autre, le sens de l'Histoire, la "vérité » à capter à l'écoute (et non, en ce cas, à la lecture) du texte produit, se coulent dans la forme toujours prégnante du vers.

9 La prose sans doute est le seul médium utilisé dans les Faits des Romains, un texte à peu près contemporain de l'Histoire ancienne jusqu'à César, et sans que son auteur justifie à un moment ou un autre les raisons de son choix. Les Faits des Romains sont pourtant un excellent exemple de la difficulté qu'ont dû avoir les nouveaux prosateurs soit à se débarrasser du vers, "la plus lourde contrainte vocale à peser encore sur la prose » selon Paul Zumthor ${ }^{11}$, soit au contraire de la tension qu'ils ont maintenu de manière concertée entre vers et prose. Pour l'auteur des Faits des Romains, styliste remarquable à défaut d'être un historien digne de foi - «son œuvre n'a rien de commun avec 
l'érudition ", déclare Paul Meyer ${ }^{12}$, mais en ajoutant aussitôt "elle n'en est pour nous que plus intéressante»- la prose narrative constitue sans doute l'ordinaire du récit. Mais, dès que surgissent la tentation ou la nécessité d'orner ce récit, ou mieux, de faire revivre l'histoire de Rome et de ses grands hommes en la contant dans une forme séduisante, en tentant de rendre "sensible aux sens" d'un lecteur médiéval une atmosphère, un milieu autres, s'insèrent alors dans la trame de la prose ordinaire formules et schèmes repris à l'écriture de la chanson de geste ou réapparaissent les techniques, codifiées par les modèles en vers, de la description, du portrait, du monologue, etc. Il serait ainsi assez facile de restituer des octosyllabes, de mettre en rime, en somme, le portrait que donne l'auteur de la trop séduisante Cléopâtre ou la somptueuse description qu'il déroule du banquet au cours duquel elle séduit César. Tout se passe donc comme si, dès qu'il faut décoller de la relation pure et simple des "faits», la prose se faisait perméable aux formules et plus encore aux cadences du décasyllabe épique ou de l'octosyllabe. Un phénomène qui s'observe aussi bien, on l'a souvent noté, chez d'autres chroniqueurs comme Villehardouin, Robert de Clari, Joinville, et que l'on peut encore nettement percevoir, vers la fin du XIV siècle, dans la Mélusine de Jean d'Arras par exemple, une prose nourrie de formules, de scènes, de motifs repris aussi bien à la chanson de geste qu'aux romans arthuriens en vers.

10 Au seuil de ce recueil, dont les différentes contributions interrogent tantôt les procédés propres à l'écriture en prose, tantôt les modalités et les enjeux des réécritures en prose de textes en vers ou encore des réécritures de prose à prose, on voudrait donc simplement rappeler combien l'adéquation prose = vérité, souvent assénée telle quelle et par les clercs médiévaux et par la critique, résiste peu à l'examen des textes. Sans doute convient-il donc de poser le problème du choix de la prose au XIII ${ }^{\mathrm{e}}$ siècle moins en termes d'une quête d'authenticité supportée/exigée par un nouveau public, ou en termes de vérité morale à dispenser (comme la vérité, la prose sortirait nue du puits), que comme la recerche neuve d'un alliage entre deux formes d'écriture dont la formule a varié selon les textes, les capacités, le métier des auteurs. Forme destinée à raconter et le plus souvent à "reconter ", la prose s'impose lorsqu'il faut "reciter ", "retraire», ou faire semblant, que la «recitation» s'appuie sur des sources existantes, généralement composées en latin (les textes à dimension historiographique) ou fantasmées (les différents livres sources des romans du Graal). Forme qui va droit devant elle, pour reprendre l'étymologie héritée d'Isidore de Séville ${ }^{13}$ la prose s'impose aussi lorsqu'il convient de suivre sans s'égarer le dévidement du temps, lorsque le récit fait le pari de l'illusion mimétique: tout dire et en bon ordre de marche, quitte à signaler, pour faire sérieux, les inévitables impasses, les passages à vide de l'action, les silences des sources ou les impuissances du narrateur. Les cadences, les formules, les motifs stylistiquement marqués de l'écriture en vers s'insinuent en revanche dès que revient en force l'exigence esthétique de l'ornementation, de l'illustration; ce que Froissart définit de manière programmatique dans le prologue de l'ultime version de ses Chroniques : «Et devés savoir que je ai ce livre cronisiet et historiiet $»^{14}$.

11 Il se peut que, dans une grande pulsion ascétique, des clercs, rompant aussi bien avec le style très travaillé des chroniques en vers $\mathrm{du} \mathrm{XII}^{\mathrm{e}}$ siècle qu'avec une prose historiographique latine souvent très ornée, très complexe et très difficile à adapter, aient cherché à réduire leur récit à des épures, à transmettre un savoir décapé de tous les vains ornements de la rhétorique La tentative est particulièrement nette dans les différentes mises en prose du Roman de Troie. On suivra avec intérêt le mouvement de 
diastole/systole qui va de la prose latine si dépouillée du pseudo-Darès à l'écriture particulièrement ornée et amplifiée du roman de Benoît de Sainte-Maure, puis fait retour aux versions rédigées en une prose minimale du XIII ${ }^{\mathrm{e}}$ siècle ou aux versions de l'histoire de Troie insérées dans l'Histoire ancienne jusqu'à César ${ }^{15}$. A dû se faire sentir, au XIII siècle comme à d'autres époques, la demande d'un savoir " compacté ", facilement mobilisable par tout lecteur désireux de s'approprier à peu de frais un pan ou l'autre du savoir. Consciencieusement limitée à la relation ordonnée des faits, l'Histoire ancienne jusqu'à César, qui a connu, à travers ses trois rédactions successives, un succès très durable, a dû exactement remplir cette attente. Mais il est clair que ce mode d'écriture n'a pas satisfait des lecteurs plus exigeants au plan artistique et que les auteurs de romans, mais aussi de chroniques (les Faits des Romains en sont un excellent exemple) ont dû assez vite « orner » leur discours et réinventer une littérarité propre à l'écriture en prose, soit en reprenant les techniques éprouvées des modèles en vers soit en inventant d'autres formules.

Il n'est pas question de revenir ici sur des points solidement établis. Ornée ou non, la prose du XIII siècle n'a jamais été une parole directement prise au piège de l'écrit, même si, précisément dans un souci de "vérité » du texte, quelques mises en scène bien connues des proses arthuriennes (Blaise ou les scribes d'Arthur mettant par écrit le texte source sous la «dictée » de Merlin ou des chevaliers rescapés des aventures), veulent en donner l'illusion. Il suffira de rappeler ici les observations formulées par Bernard Cerquiglini au début de La parole médiévale: «La prose médiévale, tout d'abord, n'est pas l'émergence en littérature de la langue de la communication, la voix qui s'élève quand on ôte la chape ou le bâillon des contraintes métriques ", le critique ajoutant peu après «Il importe donc, dans la perspective d'une théorie d'ensemble, de montrer que la prose médiévale est une élaboration consciente, et particulièrement rigoureuse, de la langue: une forme contraignante. $\aleph^{16}$ Mais les contraintes observées par le critique et les nombreuses variantes qu'elles connaissent d'un texte à l'autre - il faudrait en effet étudier, maintenant que nous disposons d'éditions d'ensemble de la plupart des grands romans arthuriens en prose, les différences repérables d'un texte à l'autre - concernent essentiellement le niveau de la syntaxe. Or il est manifeste que les prosateurs ont aussi éprouvé le besoin de se démarquer des formes de récit et non pas simplement des formes syntaxiques propres aus romans en vers et qu'ils ont institué d'autres contraintes qui concernent, elles, les macrostructures du texte produit.

La mieux connue à ce jour est sans doute la technique de l'entrelacement. D'abord repérable dans la chronique, où elle a la fonction plutôt utilitaire de faire tenir ensemble - de s'y essayer du moins - des fragments d'histoire éparpillés dans l'espace, dans les romans en prose, elle permet de substituer à l'ordre naturel du récit et du défilement du temps un ordre artificiellement complexe et qui devient de plus en plus le «lieu » où l'écrivain peut exercer ses droits sur le récit, gérer comme il l'entend, en «son bon point " précise souvent l'auteur du Tristan en prose, le texte qu'il compose. Cette technique, dont on peut suivre la genèse puis les aménagements nombreux qu'elle connaît du Lancelot en prose à Guiron le Courtois par exemple ou encore aux Prophecies Merlin, n'est cependant que la plus spectaculaire des contraintes mises au point par les prosateurs. On peut également citer/étudier à l'aune de ce très vaste corpus que compose au XIII ${ }^{e}$ siècle le roman arthurien en prose les techniques de dédoublement de personnages ou de couples, de répétition et reprise, en écho concerté, 
de motifs stylistiques et narratifs, de scènes topiques, qui expérimentent, mais à une plus vaste échelle, des procédés au reste souvent repris aux romans en vers.

Un certain scrupule, lié peut-être à l'existence d'hypotextes encore trop présents dans les mémoires des lecteurs, ou à une commune lassitude de l'écrivain et de son public pour l'artillerie lourde de la rhétorique traditionnelle explique sans doute l'absence dans les romans - les Faits des Romains, on l'a dit, procèdent tout autrement - des grandes pauses descriptives ou des portraits. Celui de Lancelot ou celui de Gauvain et de ses frères dans le Lancelot en prose représentent de ce point de vue une exception. Le texte historique en ce domaine semble plus à l'aise : on a cité, entre autres exemples, le portrait de Cléopâtre; on peut aussi citer dans les chroniques consacrées aux croisades, de la mise en prose française de l'Historia de Guillaume de Tyr à la Chronique d'Ernoul, les différentes descriptions de Jérusalem ${ }^{17}$. Mais peut-être est-ce à titre de compensation que se développe, d'abord dans des chroniques comme celle de Villehardouin, puis avec plus d'ampleur et de métier dans les romans arthuriens en prose, la pratique du discours argumentatif, à son tour drapé dans une rhétorique d'apparat, dans la forme du discours en trois points à développer canoniquement ; ou encore la pratique, qui devient un véritable stéréotype dans la Queste du saint Graal, du discours autorisé décodant pesamment des «senefiances» en général transparentes. Ces formes de discours littéraire - les modèles, en vers, s'en trouvent au reste dans les romans antiques du XII ${ }^{e}$ siècle - ne donnent pas à la prose romanesque du XIII ${ }^{e}$ siècle, une prose qui fort heureusement ne cherche de surcroît à imiter les moules phrastiques, les périodes complexes de la prose latine, comme ce sera le cas à partir du $\mathrm{XIV}^{\mathrm{e}}$ siècle, légèreté et fluidité. L'appréciation par la critique des romans et récits en prose a longtemps pâti de cette pesanteur. Mais s'il est tout à fait licite de ne pas admirer, on peut du moins mesurer l'effort entrepris et souvent réussi par les prosateurs pour diversement tordre le droit fil de la prose et retrouver peu à peu les ressources et les secrets de l'éloquence antique.

Plusieurs des études ici réunies font une large part à l'étude de la compilation, pratique clé des historiens et historiographes alors que, pour le texte romanesque, elle s'observe plutôt au niveau de la mise en cycle qu'au niveau du texte isolé, ou porte davantage sur la coexistence concertée de micro-récits de tonalité contrastée que sur la compilation proprement dite de sources diverses. On sait les connotations péjoratives qu'a conservées jusqu'à ce jour ou presque le terme de compilation sous la plume des critiques, alors qu'il s'agit d'une pratique que les auteurs médiévaux revendiquent sans complexe et qui est fondatrice de l'historiographie médiévale en langue vernaculaire. Combiner, comme le font certaines des études ici proposées, le rappel des sources avec le repérage et l'examen des ajouts et des remaniements, permet en effet de suivre au plus juste non seulement le travail d'écriture, mais les enjeux esthétiques et parfois idéologiques qui sous-tendent les fidélités et les écarts également concertés des prosateurs.

Dans La lettre et la voix. De la «littérature» médiévale, Paul Zumthor déclare à plusieurs rerpises que l'écrit, la prose tendent, «mieux que le vers ", à récuser «le présent de la voix ». Cette formulation pourrait être nuancée. La prose médiévale a assurément partie liée avec l'écrit, et en premier lieu avec cet écrit qui est presque toujours donné comme la source, réelle ou non, du nouveau texte. Avec le développement de la prose, le livre engendre le livre. Mais déjà Benoît de Sainte-Maure désigne et déclare suivre tout au long de son Roman de Troie ses sources écrites et Chrétien de Troyes, dans le 
Conte $\mathrm{du}$ Graal, se donne comme source le livre donné par le mécène... Il semble bien cependant que, dès ses premières manifestations, l'écriture en prose - une écriture à plus d'un titre totalitaire - a également pris soin de mobiliser les ressources de la voix, de la parole vive émanant d'un «je » aux diverses facettes. Dans l'ensemble des proses du Graal, il n'est guère que la tardive Estoire del Saint Graal pour se donner comme source le livre écrit par le Christ lui-même. Ailleurs, la parole de Merlin le prophète, aussitôt consignée par écrit ou gravée en forme d'inscription, il est vrai, ou mieux encore les paroles secrètes échangées entre le Christ et Joseph d'Arimathie, sont les plus solides fondations du livre à écrire, puis à lancer dans le droit chemin de la «translation». Présentée comme la forme neutre d'un récit objectif, la prose, surtout la prose du roman, s'est de fait de plus en plus ouverte aux multiples présences d'un « je » discourant, argumentant, enseignant, se moquant parfois, plus souvent encore explorant les joies et douleurs de l'amour, quitte à délaisser alors le discours prosaïque pour le chant du lyrisme.

\section{NOTES}

1.Genève, Droz, 1964.

2.0p. cit. p. 27

3.The Didot Perceval, ed. by W. Roach, Philadelphie, 1941, 1l. 1471 et ss.

4.Ed. J. Viard et E. Deprez, Paris, 1904 (SHF), p. 1-2.

5.Voir sur ce point, outre l'ouvr. cit. de Wolege-Clive, Gabrielle M. Spiegel, Romancing the Past. The Rise of Vernacular Prose. Historiography in Thirteenth-century France, Univ. of California Press, 1993.

6.Ed. C. Fahlin, t. 1 et 2, Uppsala, 1951-1954, vv. 25832-25835.

7.Voir Li Livres dou Tresor, éd. F. J. Carmody, Berkeley, 1939-1948, p. 327

8.Ed. S. Solente, T. II, Paris (SATF), 1958, vv. 8736 et ss.

9.Voir Mary Coker Joslin, The Heard Word: A Moralized History. The Genesis Section of The «Histoire ancienne »...Mississipi Romance Monographs, 1986, vv. 251-259.

10. Voir M. Szkilnik, « Ecrire en vers, écrire en prose. Le choix de Wauchier de Denain », Romania, t. 107 (1986), p. 208-230 et R. Blumenfeld-Kosinski, « Moralization and History : Vers and prose in the Histoire ancienne jusqu'à César (in B.N. fr. 20125) », Zeitschrift für Romanische Philologie, t. 97 (1981), p. 41-46.

11.Voir La lettre et la voix. De la « littérature » médiévale, Paris, Éditions du Seuil, 1987, p. 136.

12.Voir P. Meyer « Les premières compilations françaises d'histoire ancienne ", Romania, t. 14 (1885), p. 1-81.

13.Prosa est producta oratio et a lege metri soluta. Prosum enim antiqui productum dicebant et rectum. Etymologies, I, 38.

14.Ed. George T. Diller, Genève, Droz (TLF), 1972, p. 35. 
15. Voir dans cette perspective C. Croizy-Naquet, « La description de Troie et ses avatars dans le Roman de Troie en prose du XIII" siècle ", Cahiers de civilisation médiévale, t. 39 (1996), p. 303-320.

16. Voir B. Cerquiglini, «La parole médiévale. Discours, syntaxe, texte », Les éditions de minuit, 1981, p. 17 et 18.

17.Voir C. Croizy-Naquet, «La description de Jérusalem dans la Chronique d'Ernoul », Romania, t. 115 (1997), p. 69-89.

\section{AUTEUR}

\section{EMMANUĖLE BAUMGARTNER}

Sorbonne nouvelle 